\title{
Paper
}

\section{Sharpening Interpolated Image by Using Cube of Pixel Difference and Evaluation in Frequency Domain}

\author{
NAOKI $\mathrm{ONO}^{* \dagger}$ Member, KIICHI URAhAmA ${ }^{\dagger}$ Non-member
}

(Received October 20, 2015, revised February 26, 2016)

\begin{abstract}
In order to improve image resolution, some interpolation has to be applied. However, in general, an interpolation generates smooth connections between adjacent sampling points and causes blurred edges in the interpolated image. To suppress such blur in edges, it is desired that some sharpening effect is included in the interpolating process. In this paper, we propose a sharpening method using cubic operations of the differences of pixel values. Experimental results show that this method produces sharp high resolution image which is more similar than that by any other method to a target image.
\end{abstract}

Keywords: Interpolation, High resolution, Unsharp, Cubic operation

\section{Introduction}

Improving image resolution is a process for making a high resolution image from a low resolution image. In order to improve image resolution, some interpolation has to be applied. However, in general, interpolation generates smooth connections between adjacent sampling points and causes blurred edges in the interpolated image. To suppress such blur in edges, it is desired that some sharpening effect is included in the interpolating process.

For sharping images, conventional linear filters such as Laplacian operator and unsharp masking based on Gaussian filter are often used in general. A cubic unsharp masking method is proposed by G. Ramponi [1] [2]. The method uses an operator which consists of the product with Laplacian and a quadratic function of the local gradient. The cubic unsharp masking method performs effectively for the images with noise since the method suppresses the noise enhancement. However, for a noiseless image the method may not produce better result than any other sharpening method.

In order to produce a high resolution image which has extra-high frequency components, a method using cube of high pass filter is proposed [3]. In the paper, the cube of Laplacian filter is applied to video images to produce high resolution images of them and the validity of the method are shown for producing high frequency components.

In this paper, we propose a sharpening method which is not cube of linear filter but uses weighted sum of cubic operations of the differences between a value of pixel and values of the surrounding pixels. Experimental results for an interpolated image show that this method produces a sharp high resolution image which is more similar to a target image than that by any other sharpening method.

\footnotetext{
* Corresponding: ono@design.kyushu-u.ac.jp

Faculty of Design, Kyushu University, Shiobaru 4-9-1, Minami-ku, Fukuoka Japan 815-8540
}

\section{Sharpening for interpolated image}

2.1 Unsharp masking by liner operation The fundamental idea of unsharp masking is to add high frequency components to the input image. The high frequency components of an input image is obtained by subtraction of a low pass filtered version of the input image from the input image itself. In other words, the input image is modified by its own high frequency component.

Given interpolated image $\left\{d_{i j}(i=1, \cdots, M ; j=1, \cdots, N)\right\}$, an unsharp masked image $f_{i j}$, which is derived by the standard unsharp masking with linear low pass filter, is given by

$$
f_{i j}=d_{i j}+\delta\left(d_{i j}-\tilde{d}_{i j}\right),
$$

where $\tilde{d}_{i j}$ is a low pass filtered version of $d_{i j}$.

For making a low pass filtered image $\tilde{d}_{i j}$, using Gaussian filter

$$
w_{l m}=\frac{e^{-\alpha\left(l^{2}+m^{2}\right)}}{\sum_{l=-p}^{p} \sum_{m=-p}^{p} e^{-\alpha\left(l^{2}+m^{2}\right)}},
$$

the linear unsharp masking (LUM) produces an enhanced image $f_{g i j}$ described by

$$
\begin{aligned}
f_{g i j} & =d_{i j}+\delta\left(d_{i j}-\tilde{d}_{i j}\right) \\
& =d_{i j}+\delta\left(d_{i j}-\sum_{l=-p}^{p} \sum_{m=-p}^{p} w_{l m} d_{i+l, j+m}\right) \\
& =d_{i j}+\delta \sum_{l=-p}^{p} \sum_{m=-p}^{p} w_{l m}\left(d_{i j}-d_{i+l, j+m}\right),
\end{aligned}
$$

where $\alpha(>0)$ and $p$ are parameters which smoothness and size of mask respectively. Furthermore we control the effect of sharpening by a parameter $\delta(>0)$. 
2.2 Sharpening by cube of liner masking In the process of unsharp masking, if we adopt the cube of the difference given by the subtraction of a low pass filtered version from the input image as the high frequency components, slight changes in the image can be enhanced and detail information becomes more visible effectively. According to this idea, an enhanced image $f_{c i j}$ is produced by

$$
\begin{aligned}
f_{c i j} & =d_{i j}+\delta\left(d_{i j}-\tilde{d}_{i j}\right)^{3} \\
& =d_{i j}+\delta\left(d_{i j}-\sum_{l=-p}^{p} \sum_{m=-p}^{p} w_{l m} d_{i+l, j+m}\right)^{3} \\
& =d_{i j}+\delta\left(\sum_{l=-p}^{p} \sum_{m=-p}^{p} w_{l m}\left(d_{i j}-d_{i+l, j+m}\right)\right)^{3} .
\end{aligned}
$$

The second term in eq. 4 is the cube of LUM and the input image $d_{i j}$ is modified by the cube of LUM. Using the cube of Laplacian instead of second term in eq. 4 as the high frequency component, the sharpening procedure is equivalent to that in [3].

\subsection{Sharpening by using cube of pixel differences}

We propose a sharpening method which is not cube of linear filter but uses weighted sum of cubic operations of the differences between a value of pixel and values of the surrounding pixels, i.e.

$$
f_{d i j}=d_{i j}+\delta \sum_{l=-p}^{p} \sum_{m=-p}^{p} w_{l m}\left(d_{i j}-d_{i+l, j+m}\right)^{3} .
$$

This method also enhances slight changes effectively. However, since the value of modification in eq. 5 often becomes extremely large, we suppress the excessive modification by setting a limit value $\mathrm{L}$. Needless to say, the limitation should be applied to the cube of LUM in eq.4.

\section{Experiments}

3.1 Sharpening image An original image, shown in Figure 1, consists of $256 \times 256$ pixels with 256 gray levels. Firstly, we interpolated the original image into an enlarged image which consists of $512 \times 512$ pixels by the bilinear interpolation. Figure 2 shows the interpolated image. Applying the three sharpening methods described above to the interpolated image, we had three enhanced images. In the experiment, we set the parameters $p=2, \alpha=0.5, \delta=0.4$ and $L=4$.

We compared the proposed method with a conventional LUM and the cube of LUM experimentally. Figure 3, Fig. 4 and Fig. 5 show improved images by the LUM, the cube of LUM and the proposed method respectively. The image by the proposed method shows clear details of textures with suppressing excessive edge enhancement such as overshoot and undershoot. Although the LUM also gave clear texture details, all edges are enhanced too much. The cube of LUM lost the texture details especially in the area of a hat.

3.2 Comparison on the similarities of local complexity In general, a performance of image processing is evaluated by using PSNR. However PSNR does not always show a reasonable value especially on sharpness of images. Since we now focus on the sharpness and enhancement of image details, we use the local complexity to evaluate the performance.

We employ the local standard deviation as the local complexity. Assuming that the local standard deviation of an enhanced image and that of the target image are represented by $s_{i j}$ and $s_{0 i j}$ respectively, the sum of difference of local complexities between the enhanced image and the target image

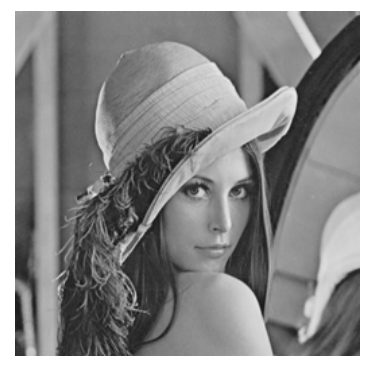

Figure 1: An original image.

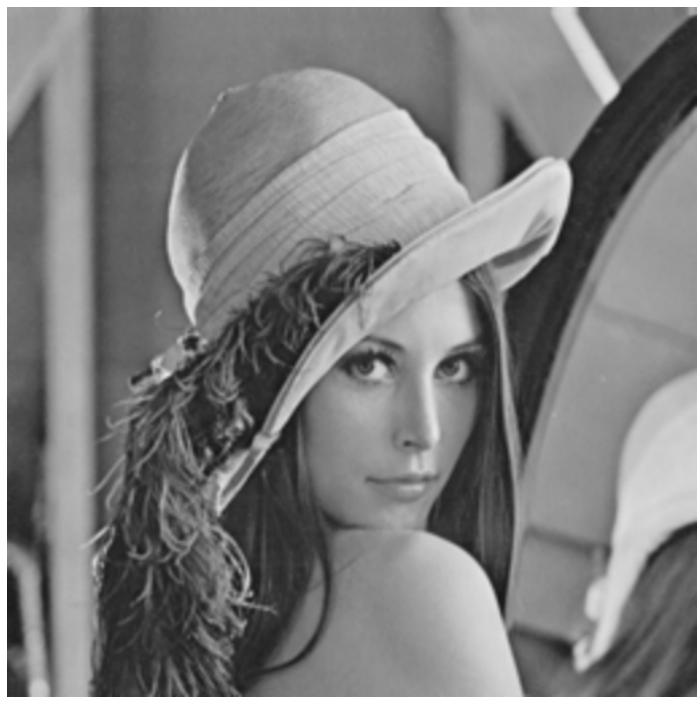

Figure 2: Bilinear interpolated image.

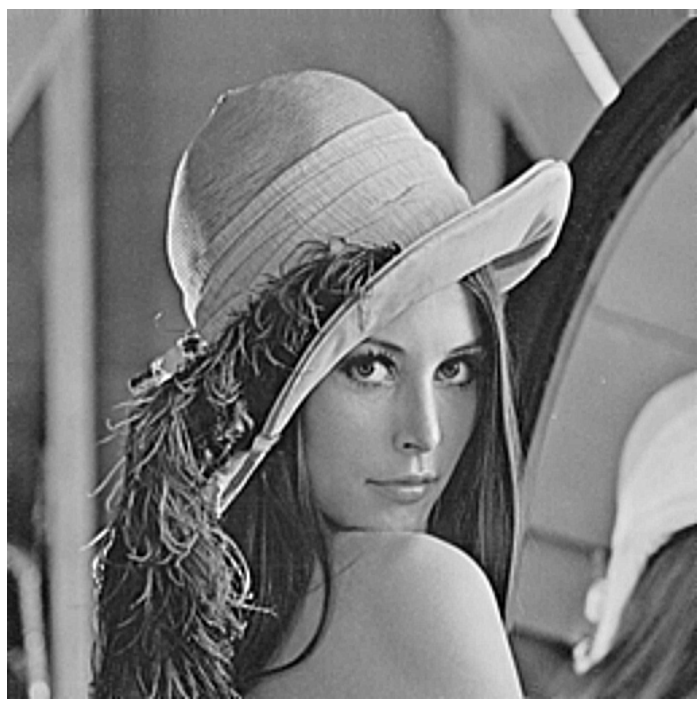

Figure 3: Result by the LUM. 


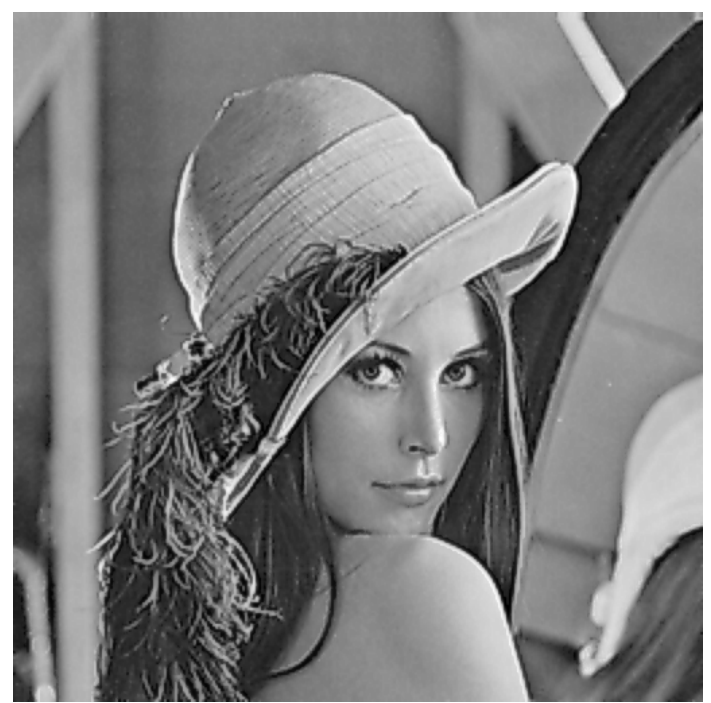

Figure 4: Result by the cube of LUM.

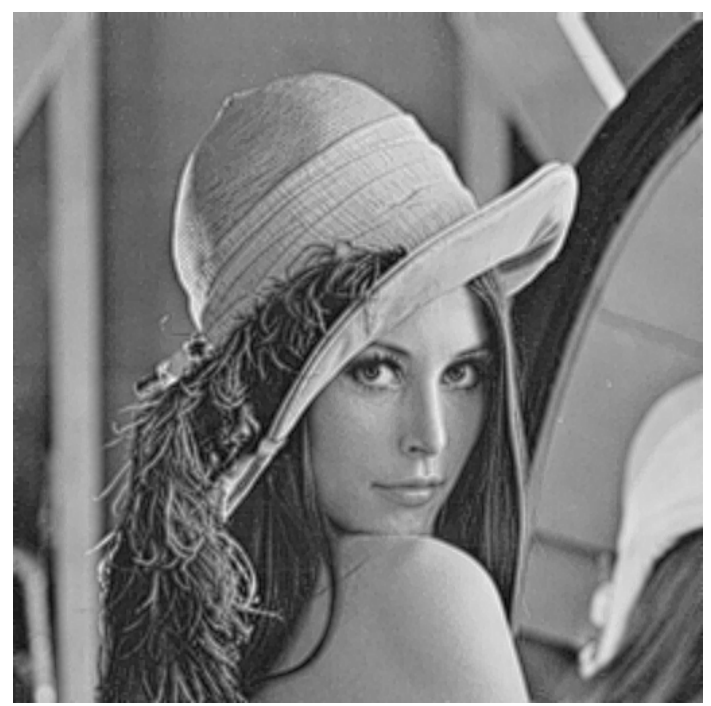

Figure 5: Result by the proposed method.

is obtained by

$$
S_{d}=\frac{1}{M N} \sum_{i=1}^{M} \sum_{j=1}^{N}\left(s_{i j}-s_{0 i j}\right)^{2}
$$

The value derived by Eq. 6 gives the similarity between an enhanced image and a target image in the meaning of local complexity. The smaller $S_{d}$ is, the more similar the enhanced image and the target image are. In this experiment, we used a high resolution $512 \times 512$ image shown in Fig. 6 as the target image, where this image is the true high resolution image of the input $256 \times 256$ image. Standard deviations $s_{0 i j}$ for the target image and $s_{i j}$ for created images shown in Fig. 3, 4, 5 are calculated with $7 \times 7$ square window.

Table 1 shows the sum of difference of local complexities between the enhanced image and the target image by Eq.6. $S_{d}$ for resultant images by the LUM and the cube of LUM are larger than $S_{d}$ for the bilinear interpolated image which is before enhancement. Especially the difference

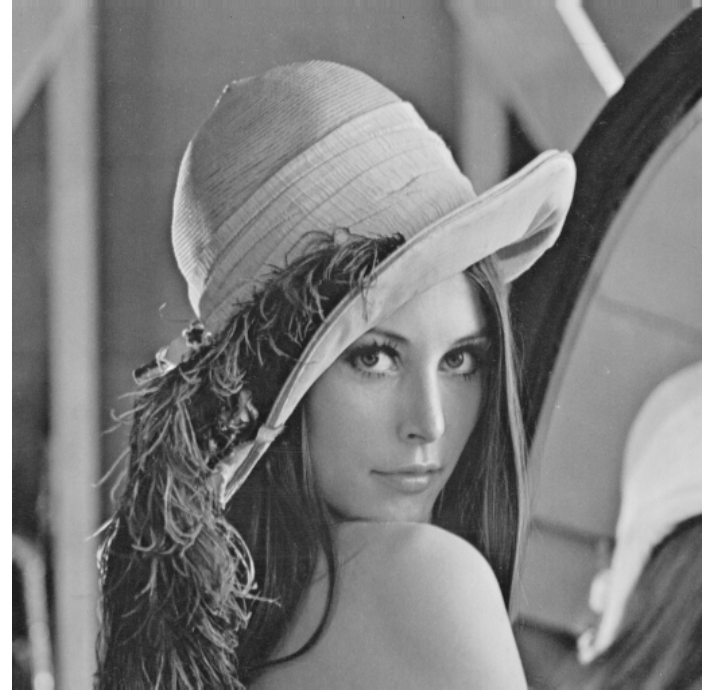

Figure 6: A target image.

Table 1: The similarities of local complexity.

\begin{tabular}{cc}
\hline & $S_{d}$ \\
Bilinear interpolation & 12.1485 \\
LUM $\alpha=0.5, \delta=0.4$ & 42.2887 \\
Cube of LUM $\alpha=0.5, \delta=0.4, L=4$ & 27.2918 \\
Proposed method $\alpha=0.5, \delta=0.4, L=4$ & 6.1270 \\
\hline
\end{tabular}

of the LUM is much larger. These results mean that the LUM and the cube of LUM degraded the similarity of local complexity for the bilinear interpolated image. Sd for the proposed method is smallest in this experiment. This shows that the proposed method improved the interpolated image and gave a most similar image to the target image at the point of complexity.

3.3 Comparison in the frequency domain By improving the resolution of image, the components in higher frequency domain are newly created. We evaluate the components in the low frequency domain included in the original low resolution image and those in the high frequency

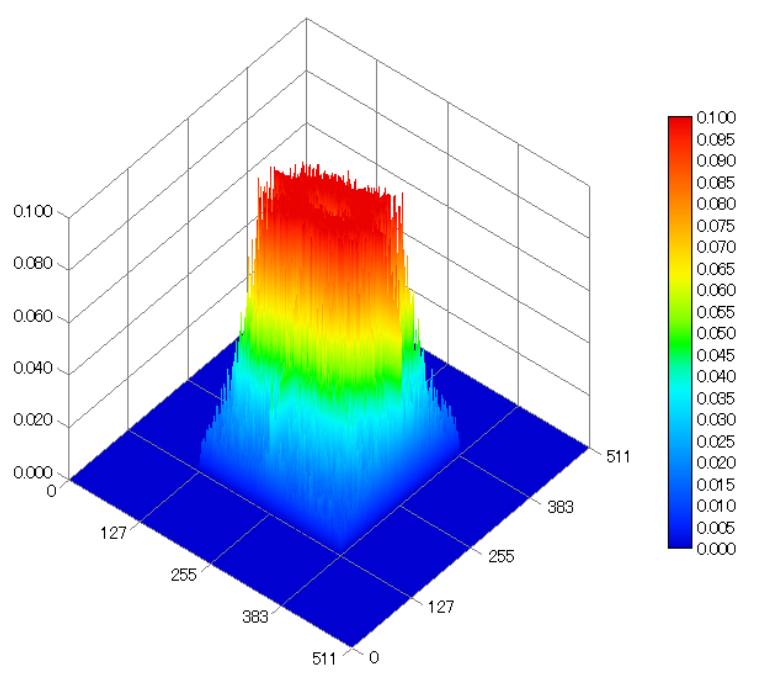

Figure 7: Power spectrum of the original image. 


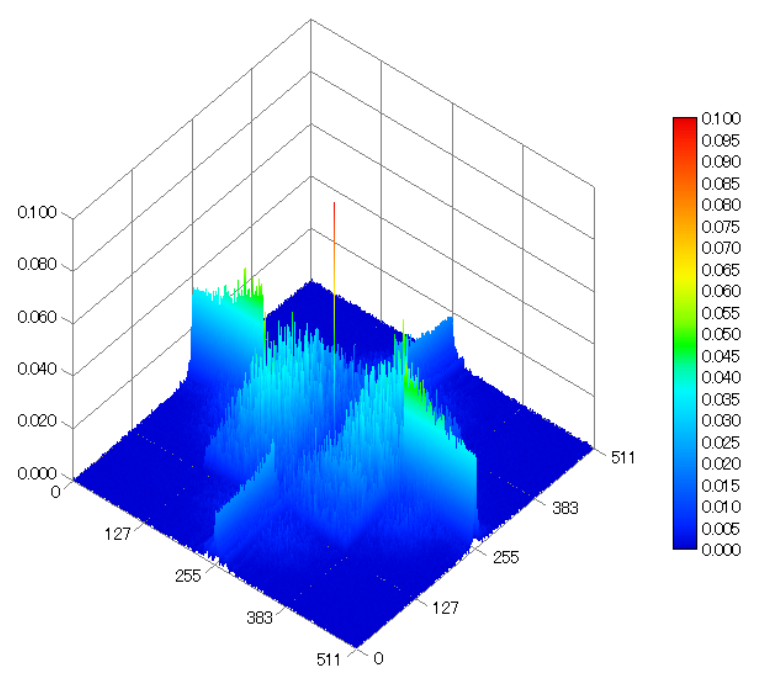

Figure 8: Difference of power spectrum between a bilinear interpolated image and the original image.

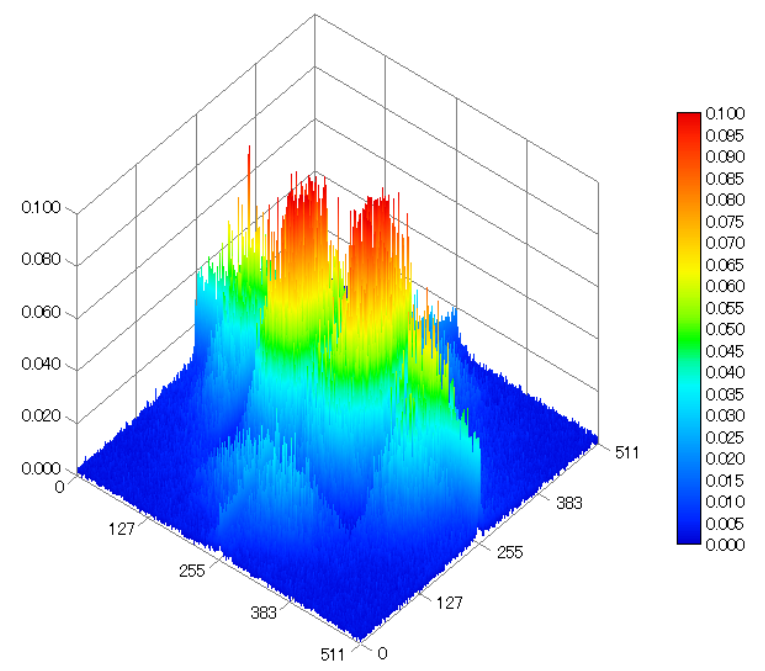

Figure 9: Difference of power spectrum between an LUM image and the original image.

domain generated by the interpolation respectively.

After improving the image, it is desired to preserve the frequency components included in an input image before interpolation in order not to change the original image information. On the other hand, the high frequency components which is not contained before interpolation should be create effectively to give a better impression as a high precision image.

Figure 7 shows the FFT result of the original low resolution image. Although the frequency domain of the original image is $256 \times 256$, the components are superimposed in the domain of $512 \times 512$ to compare with the interpolated high resolution images. Figure 7 also shows the original image does not have high frequency components in the outside of $256 \times 256$ low frequency domain. In the graph the values over 0.01 are truncated in order to use the same scale in the following graphs (Fig.8, 9, 10 and 11).

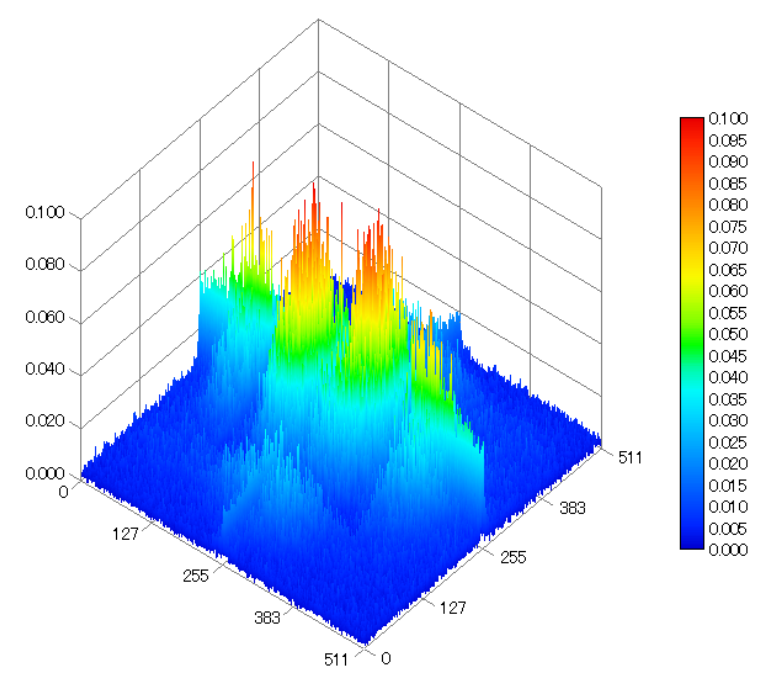

Figure 10: Difference of power spectrum between a cube LUM image and the original image.

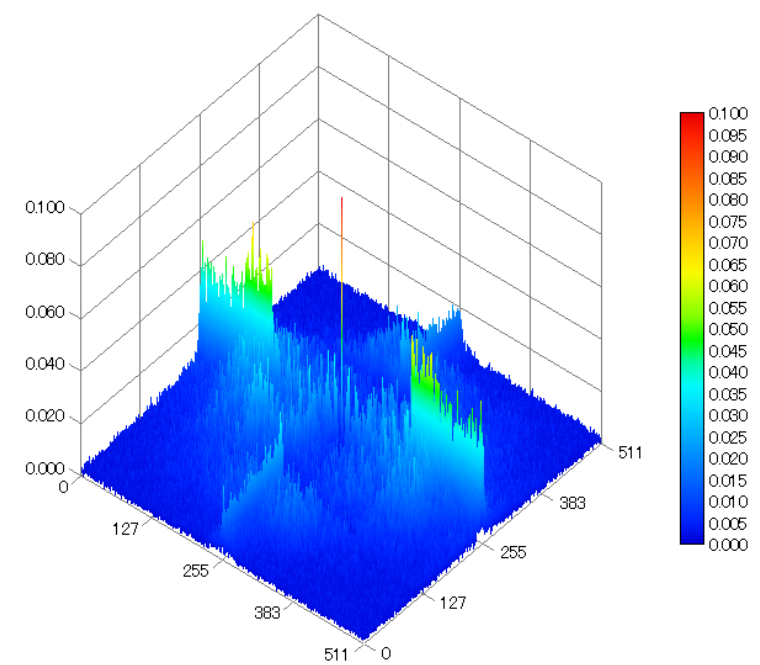

Figure 11: Difference of power spectrum between an image by the proposed method and the original image.

Figure 8 shows the difference of frequency components between the bilinear interpolated image shown in Fig.2 and the input low resolution image. Needless to say, difference in the outside of $256 \times 256$ is newly created high frequency components. Figure 9, 10, 11 show the differences between frequency component of Fig.2 and those of Fig. 3, 4, 5 respectively. We evaluated whether the method creates high frequency components with suppressing the change of components in $256 \times 256$ low frequency domain.

Although the bilinear interpolated image has new high frequency components which look like thin walls in Fig.8, these components are yielded by the linear interpolation process and are not created from the characteristic of the input image.

From Fig.9 and 10, although enhanced images by the LUM and the cube of LUM have high frequency components, components in low frequency domain have changed 
remarkably. This shows that the information of original image was changed obviously. Furthermore, the differences in low frequency domain for resultant images by the LUM and the cube of LUM are larger than those for the bilinear interpolated image which is before enhancement. This means that the LUM and the cube of LUM distorted the original information from the bilinear interpolated image.

Figure 11 shows that the result by the proposed method have high frequency components and the difference of components in $256 \times 256$ low frequency domain are smaller than those of other resultant images. From this experiment, the proposed method yields high frequency components with suppressing change of information of original image reasonably.

\section{Conclusions}

In this paper, we proposed a sharpening method which is not cube of linear filter but uses weighted sum of cubic operations of the differences between a value of pixel and those of the surrounding pixels.

We applied some enhancement procedures for a linear interpolated image and compared the similarities of local complexity of the resultant images and a target image experimentally. The proposed method improved the interpolated image and gave most similar image to the target image at a point of complexity. We also evaluated the resultant images in the frequency domain. The proposed method yielded high frequency components with suppressing change of information of the original image reasonably.

Experimental results for an interpolated image show that the proposed method produces sharp high resolution image which is more similar to a target image than that by any other method.

\section{References}

[1] G. Ramponi, "A cubic unsharp masking technique for contrast enhancement", Signal Processing, Vol.67, pp.211-222, 1998.

[2] A. Polesel, G. Ramponi, V. J. Mathews, "Image enhancement via adaptive unsharp masking”, IEEE Trans. on Processing, Vol.9, No.3, pp.505-510, 2000.

[3] S. Ghoshi, "A new signal processing method for video", MMSys ' 12 Proceedings of the 3rd Multimedia systems Conference, pp.47-52, 2012.

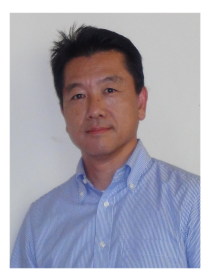

Naoki Ono (Member) was born in Saga, Japan, in 1961. He received a Ph.D. degree in engineers from Kyushu University in 1997, and is presently an associate professor at Kyushu University. He has worked on image processing and pattern recognition. He is member of IIAE and IEICE.

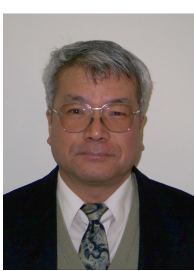

Kiichi Urahama (Non-member) received a $\mathrm{Ph} . \mathrm{D}$. degree in engineering from Kyushu University, and is presently a professor at Kyushu University. He has worked on image processing and pattern recognition. He is member of IEICE. 